\title{
Masking Conventional Metallic Cast Post for Enhancing Esthetics
}

\author{
${ }^{1}$ Naisargi P Shah, ${ }^{2}$ Amit Mahadeo Gaikwad, ${ }^{3}$ Sabita M Ram, ${ }^{4}$ Jyoti B Nadgere
}

\begin{abstract}
Loss of tooth structure occurs due to caries, endodontic treatment, or fracture caused by trauma; restoring these teeth is difficult task for a clinician. Post and core is an option for teeth when excessive tooth structure is lost and teeth need to be restored with crown and bridge procedure. They can be prefabricated post with composite built up or a one-piece custom-made post. The custom-made cast metal post and core have disadvantage due to their color that imparts grayish hue to overlying ceramic restorations. Masking the metal core can enhance the esthetic of all ceramic restorations that are placed over the cast post and core. A case report is being presented where the metallic core was masked with ceramic built up on labial surface of metal core.
\end{abstract}

Keywords: All ceramic restorations, Lithium disilicate, Metal cast post, Post and core.

How to cite this article: Shah NP, Gaikwad AM, Ram SM, Nadgere JB. Masking Conventional Metallic Cast Post for Enhancing Esthetics. J Contemp Dent 2016;6(1):85-89.

Source of support: Nil

Conflict of interest: None

\section{INTRODUCTION}

Post and core are regarded as the foundation restoration for mutilated dentition. ${ }^{1}$ Post is required for retention of core and reinforcement of tooth structure. For post and core to be successful, a ferrule of $2 \mathrm{~mm}$ is mandatory; this can be achieved by preservation of remaining tooth structure, crown lengthening, or orthodontic extrusion. $^{2}$

The post and core can be prefabricated post with composite built-up core or a one-piece custom-made post and core that can be in metal or zirconia material. ${ }^{3}$ The custom-made post and core are indicated in situations wherein gross tooth structure is lost, teeth having wide canals, anterior deep bite, and where a change in angulation is required for enhancing esthetic by repositioning of restoration in the arch. ${ }^{3}$

\footnotetext{
${ }^{1,3,4}$ Professor, ${ }^{2}$ Postgraduate Student (3rd Year)

${ }^{1-4}$ Department of Prosthodontics, Crown and Bridge, MGM Dental College and Hospital, Navi Mumbai, Maharashtra, India

Corresponding Author: Naisargi P Shah, Professor Department of Prosthodontics, Crown and Bridge, MGM Dental College and Hospital, Navi Mumbai, Maharashtra, India, Phone: +919819238483, e-mail: drnaisargi69@gmail.com
}

The zirconia post and core may be used for their esthetic outcome but are more expensive and difficult to fabricate. ${ }^{4}$ A metal post and core is used routinely in practice; however, its esthetic outcome when placed under all ceramic restorations is not acceptable due to the grayish hue imparted to the final restoration. The labial surface of the metal core can be masked with ceramic layer after the casting of cast post and core. This will enhance the outcome of the final all ceramic restorations and give a successful esthetic result.

A case wherein anterior fracture teeth were restored with a masked metal core and all ceramic crown and bridge is presented in this article.

\section{CASE REPORT}

A 28-year-old male patient reported to the Department of Prosthodontics with the chief complaint of dislodged anterior crowns, swelling, and pain in upper anterior region. He desire relief of pain and improvement of esthetic. Past dental history revealed that the patient had undergone root canal treatment with 11, 12, and 21; periapical surgery was done with 11 and 12 and restored with porcelain fused to metal crowns. The crowns along with the prepared teeth were dislodged. Family, medical, and personal history was not relevant.

Extraoral examination showed that the patient had square facial form and flat facial profile due to loss of anterior teeth. The lip length was average. On smiling, a space of anterior missing teeth was visible (Fig. 1).

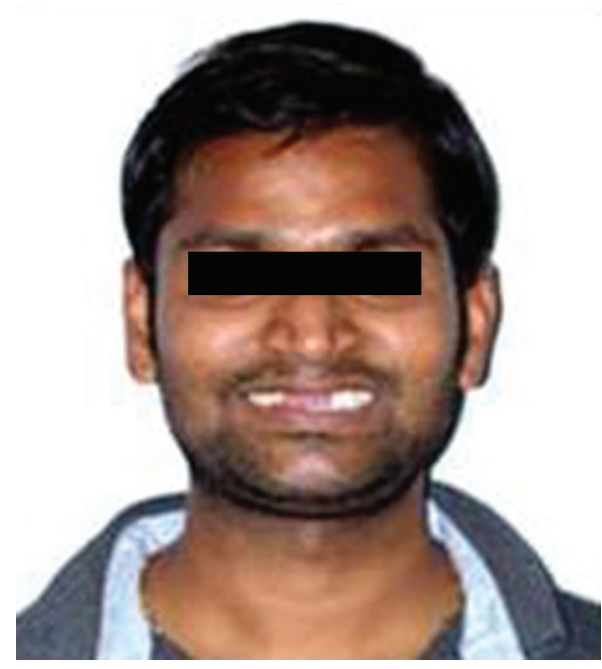

Fig. 1: Extraoral 


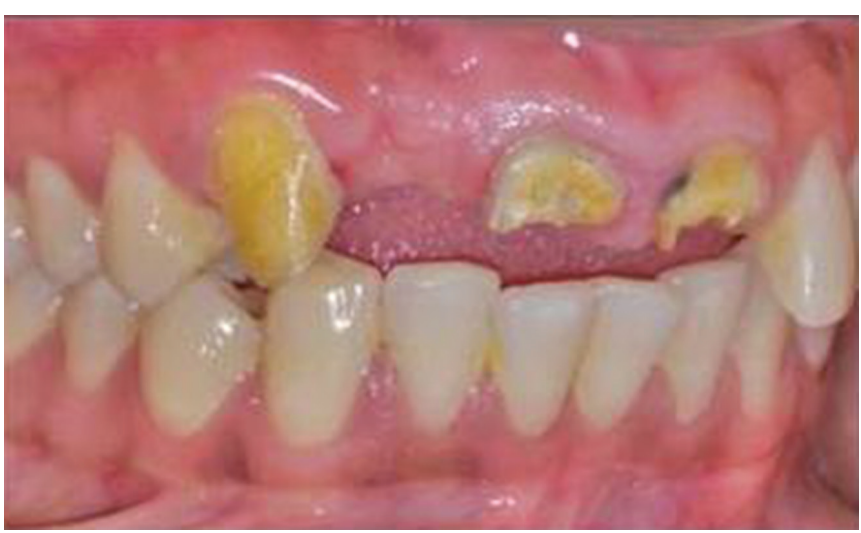

Fig. 2: Intraoral

Intraoral examination showed missing 12. Fracture with 11 and 21 was till gingival level. Intraoral sinus with 11 and pain on percussion was positive with 11 and 21. Caries was seen with 26, 36, 37, 46, and 47 . The patient had poor oral hygiene along with gingival inflammation in relation to upper anterior region (Fig. 2). The edentulous area with 12 showed loss of vertical height that would make it difficult to place an esthetic pontic.

Intraoral periapical radiographic examination showed incomplete obturation with 11 and 21 and large periapical lesion with 11.

Study models were made from diagnostic impression and analyzed for determining treatment plan for anterior all-ceramic restoration. A diagnostic mock-up was done on the study model and putty index was prepared. A mock-up trail was done directly into patient mouth using the putty index and Protemp for diagnostic planning of final restoration and approval of patient was taken on esthetic outcome of the provisional restoration prior to planning the other procedure.

A multidisciplinary treatment was planned for the patient that involved endodontic treatment, periodontic management, and prosthetic rehabilitation. Following treatment was carried for the patient.

Scaling and polishing was done for both the arches. Preoperative radiograph showed incomplete obturation with 11 and 21 and large periapical lesion with 11; therefore, root canal treatment was repeated with 11 and 21 and periapical surgery was carried out with 11 . Intentional root canal treatment was carried out with 13 , as adequate tooth structure was not available for restoration and tooth preparation would have led to exposure of pulp as the pulp horn was large. Provisional restoration with ovate pontic design was made with the help of putty index. Ridge defect was observed in relation with 12 that would have affected final esthetic outcome. So, modified Abrahams technique was followed to overcome the ridge defect and to achieve the desired emergence profile. ${ }^{5}$ In this method, a de-epithelialized

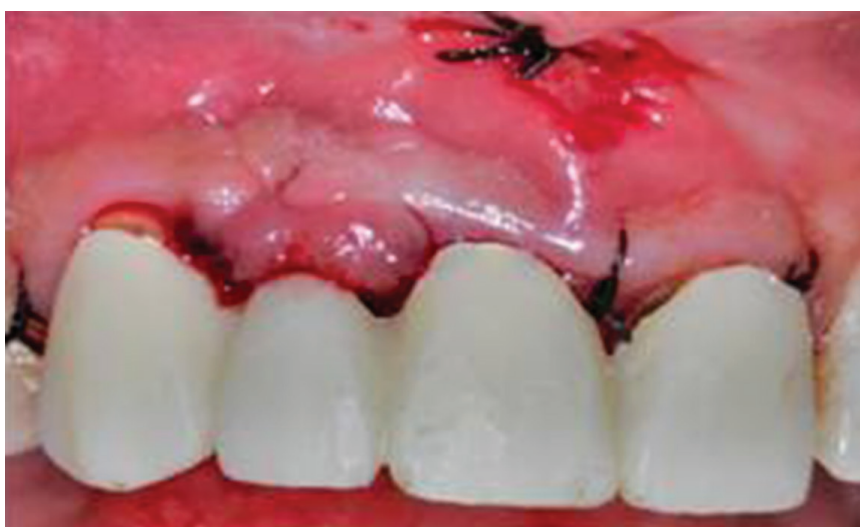

Fig. 3: Provisional restoration with ovate pontic design placed after surgery

palatal flap was dissected and a pedicle was displaced toward the buccal aspect of the ridge. The connective tissue was then rolled below the buccal flap in the area of the deformity in order to correct buccolingual ridge defect. ${ }^{5}$ Crown lengthening was simultaneously carried out with 11 and 21 to achieve adequate ferrule. Provisional restoration, which was already fabricated, was cemented immediately after the surgery. Patient was on provisional restoration for 6 months for the development of pontic site (Fig. 3).

An all-ceramic restoration was planned for the patient, as he had high esthetic demand. Shade selection was done using spectrophotometer by placing it on adjacent tooth and shade was noted. ${ }^{6}$ Tooth preparation was done to achieve the desired ferrule. Indirect technique was followed for cast postfabrication. ${ }^{3}$ A custom-made resin post was fabricated to fit the canal two-third its length and picked up in an irreversible hydrocolloid impression material (Figs 4 and 5).

Wax patterns were fabricated with casting wax, casted, and finished. Their fit was checked on the master cast and intraoral trail of cast post was done (Fig. 6). Radiograph was taken to determine the length and fit of the cast post (Fig. 7). Metal on the labial surface was removed to achieve space for masking material and

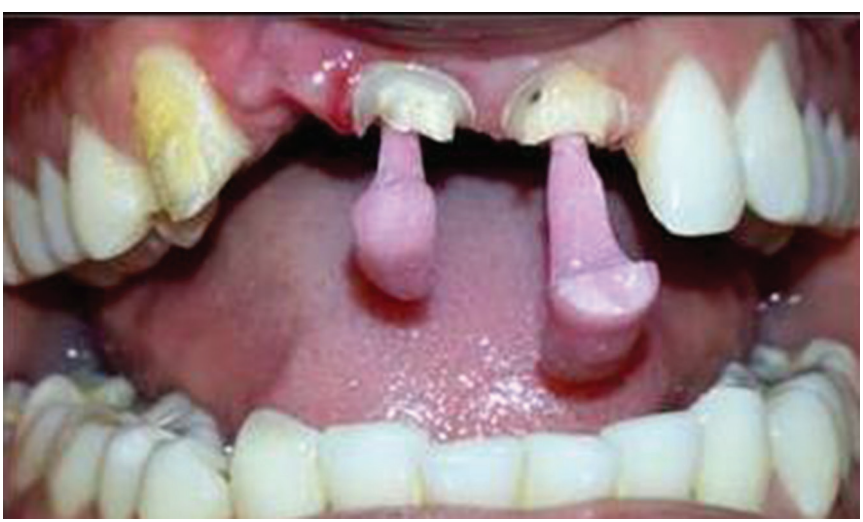

Fig. 4: Acrylic post into the canal 


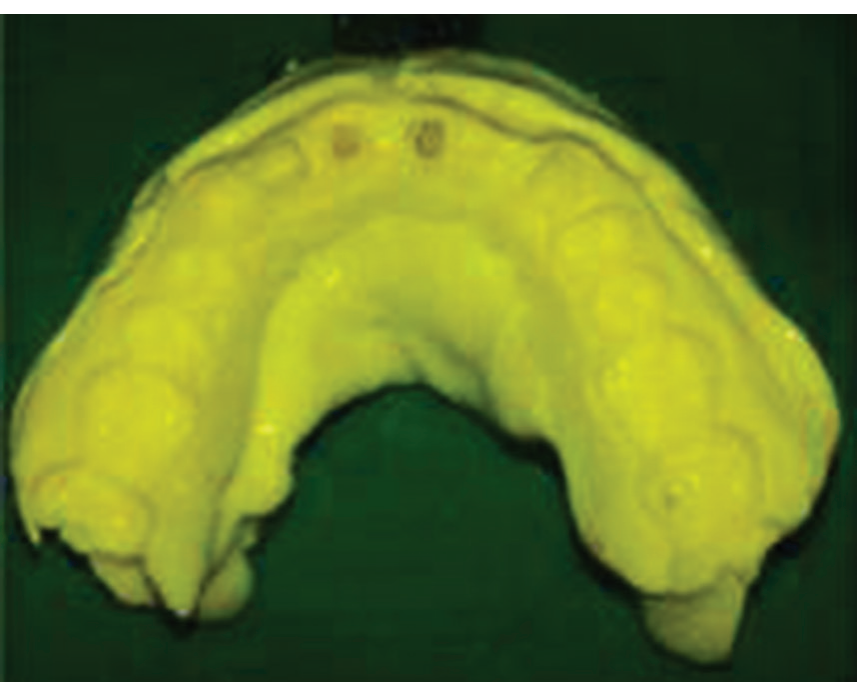

Fig. 5: Impression for cast post fabrication

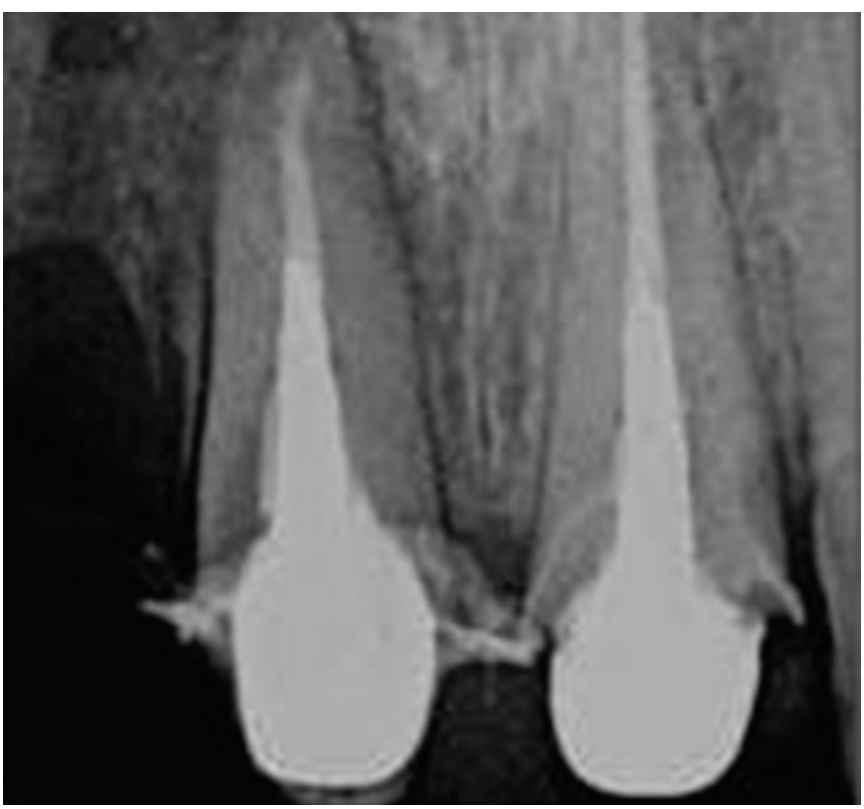

Fig. 7: RVG before cementation of cast post

sandblasted with aluminum oxide powder to achieve rough surface. Ceramic opaquer was fired on it to mask the color of the metal (Figs 8 and 9).

Cementation of cast post was done with zinc phosphate cement. ${ }^{7}$ Final impression was made with addition silicone in two stages and cast was poured in Type IV gypsum for the fabrication of lithium disilicate crown and bridge. The wax pattern planned for the lithium disilicate restoration replicated the provisional restoration in its form specially the pontic that was an ovate pontic contoured well to fit the developed edentulous site. Final crown and bridge was fabricated by pressed technique using lithium disilicate ingots. The bisque trail was done to determine the fit, esthetic, and occlusion of the crown and bridge restoration. It did not reveal the underlying unesthetic metal because of the

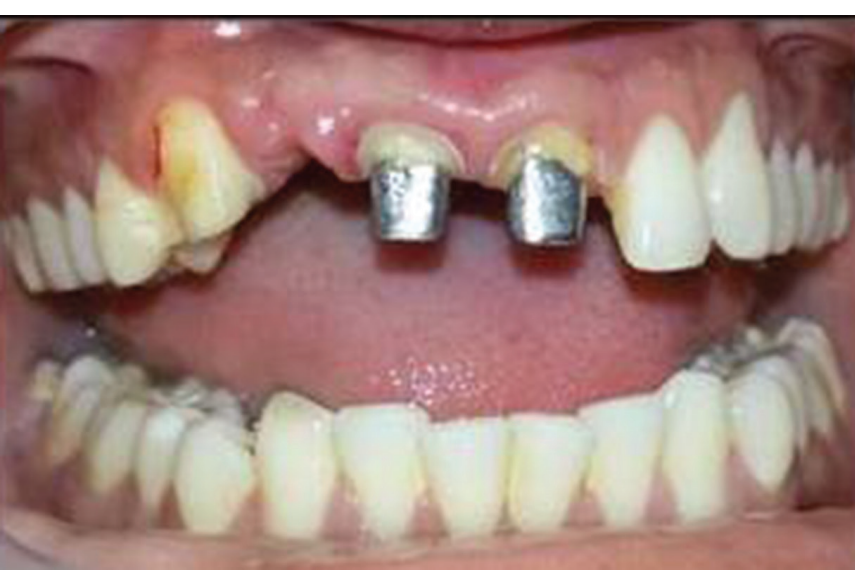

Fig. 6: Intraoral trial of cast post

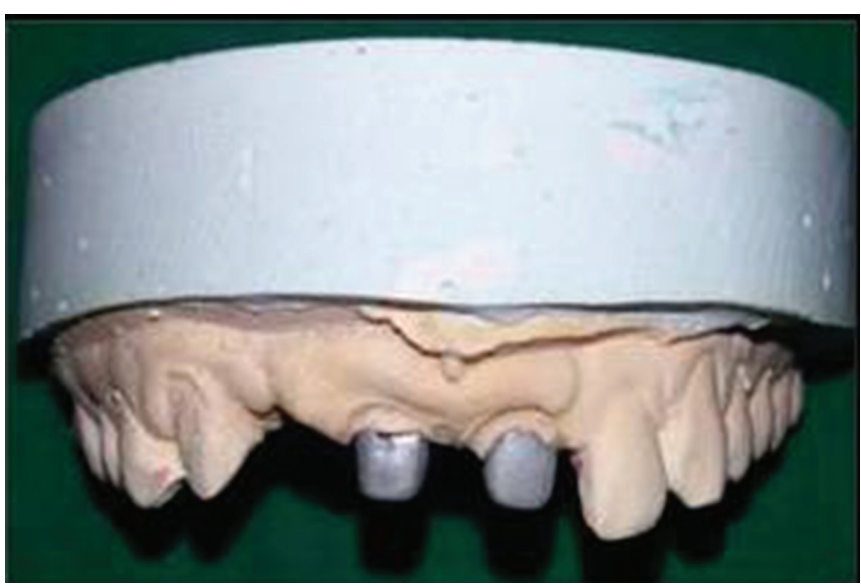

Fig. 8: Cast post and core after sandblasting

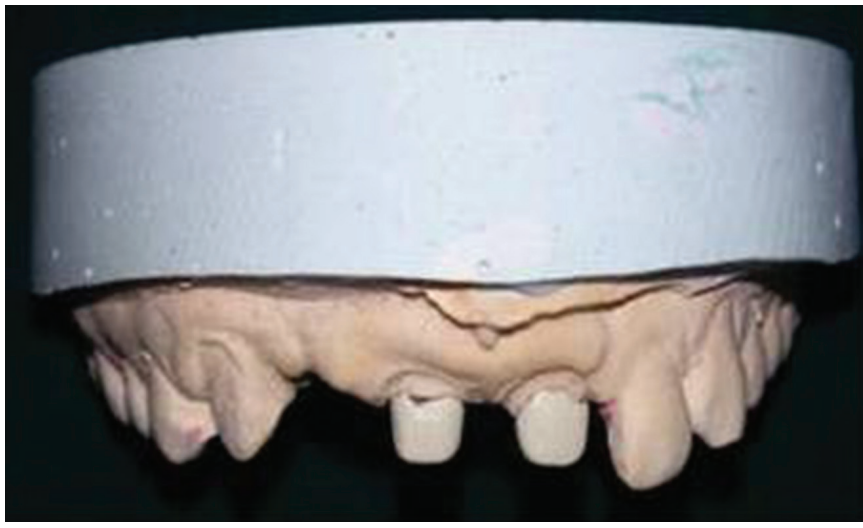

Fig. 9: Cast post and core after application of ceramic opaquer

masked metal core. The final glazing of the restoration was carried out and was ready for cementation.

For cementation procedure, etching of ceramic was carried out with $10 \%$ hydrofluoric acid for 15 seconds (Fig. 10). Simultaneously, intraorally canine and remaining tooth structure was etched with $37.5 \%$ of orthophosphoric acid for 15 seconds, as the preparation was in dentin (Fig. 11). Metal core of cast post was not etched, as it was rough. Etched surface was thoroughly washed with water and dried. It showed white frosted appearance 
of both tooth and ceramic. Silane application was done on ceramic; simultaneously, bonding agent was applied on canine and remaining tooth structure. It was light cured for 10 seconds. Final crown and bridge cementation

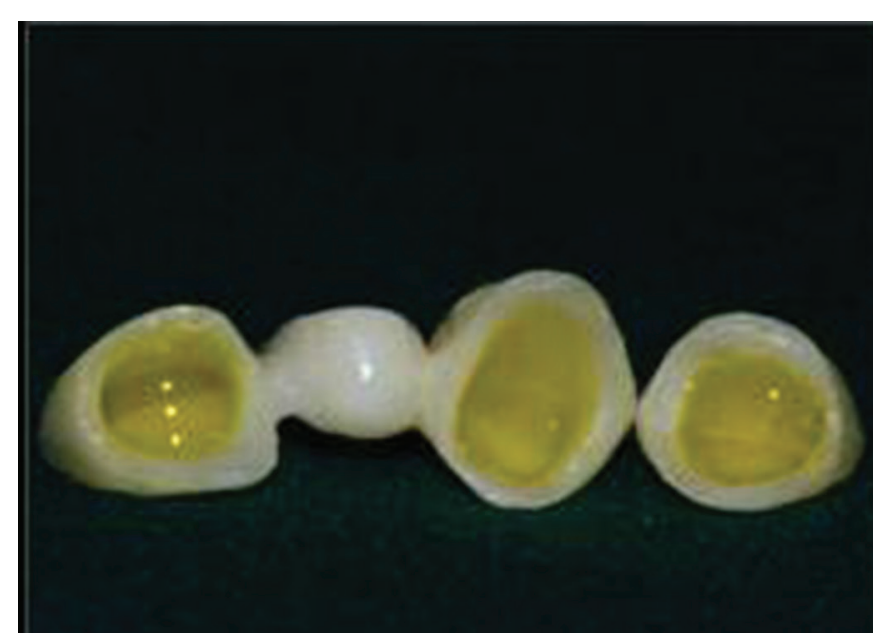

Fig. 10: Silane application

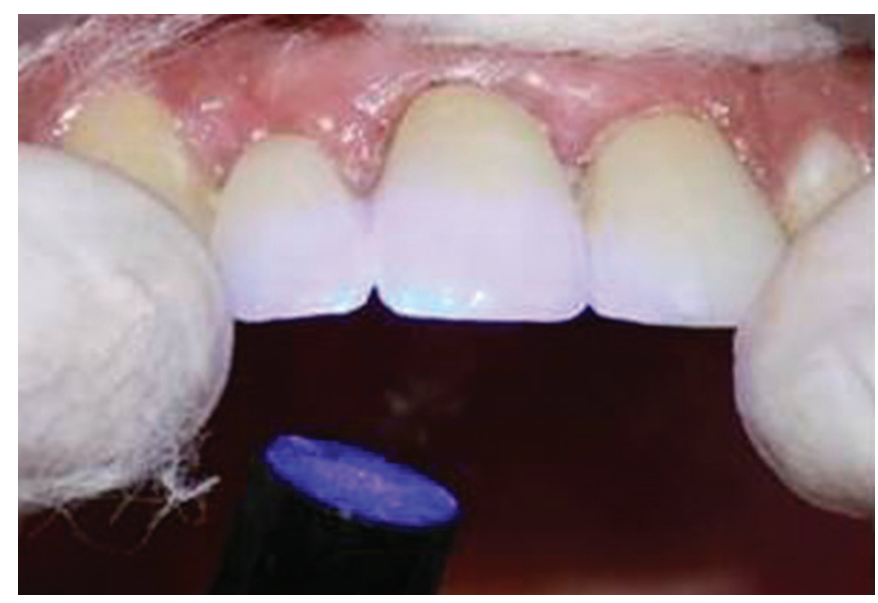

Fig. 12: Light curing of dual-cure resin cement

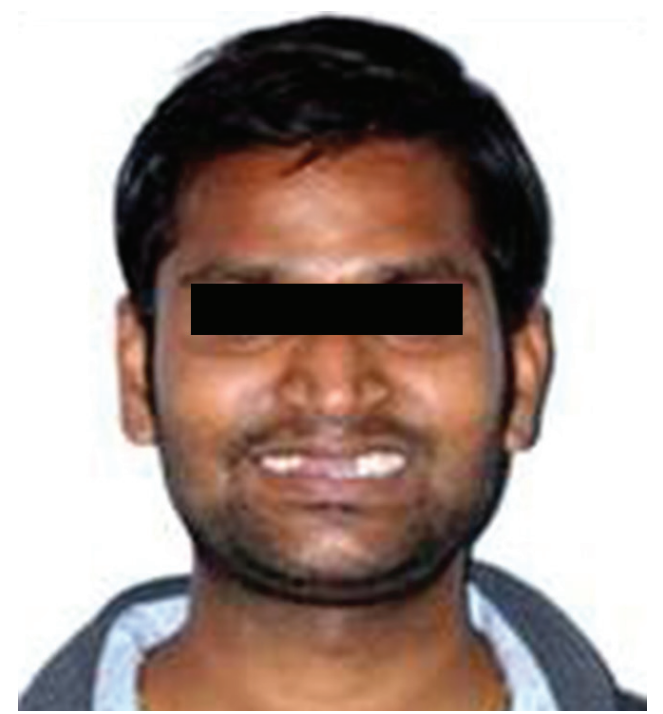

Fig. 14: Preoperative extraoral was done with dual cure resin cement using Rely X U 200 (Fig. 12). Patient was happy and satisfied with the treatment (Figs 13 to 15). Patients recall was done after 24 hours, 1 week, 1 month, and 3 months.

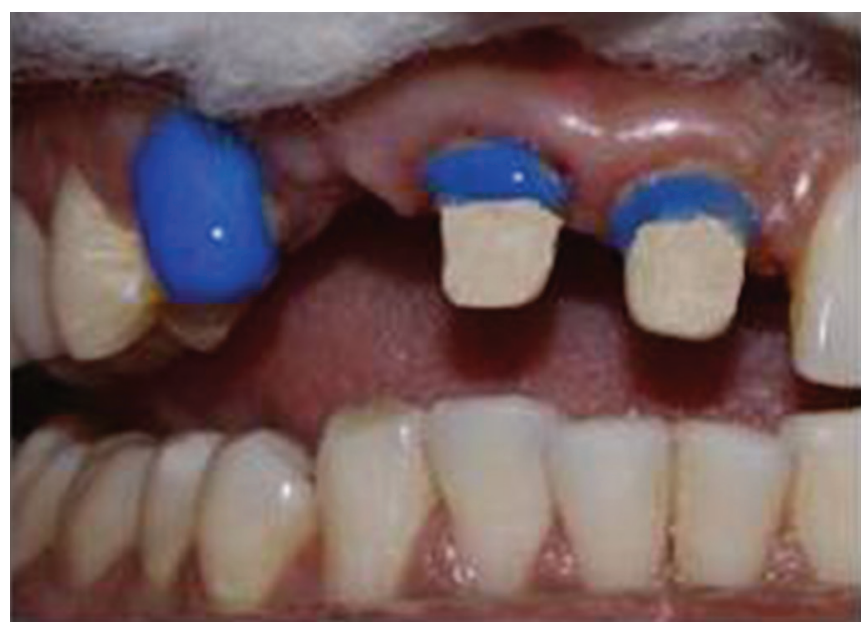

Fig. 11: Etching of tooth with orthophosphoric acid

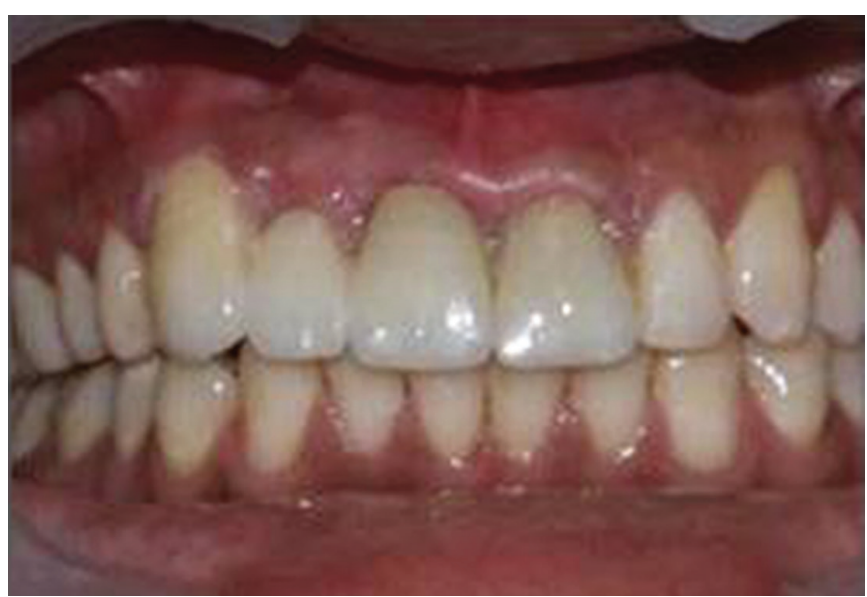

Fig. 13: Postoperative intraoral

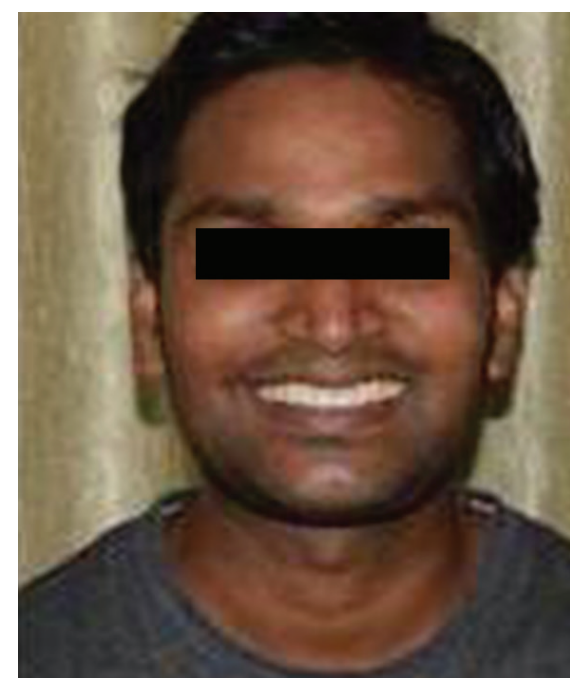

Fig. 15: Postoperative extraoral 


\section{DISCUSSION}

Gross destruction of teeth poses a restorative challenge for the clinician. Post and core restoration are boon for managing such teeth. For post and core restoration, at least $2 \mathrm{~mm}$ of ferrule is essential. ${ }^{3}$ In this case, the remaining tooth structure of both central incisors was inadequate for post and core restoration. Orthodontic extrusion require long time period, so crown lengthening was carried out in 11 and 21 to achieve desire ferrule.

Selection of post depends on several factors, namely the amount of remaining tooth structure, position of teeth, occlusion of patient, and many others. The masked metal cast post was suggested as the treatment option for 11 and 21, as the amount of remaining tooth structure was inadequate to restore with prefabricated post and composite core built up. Zirconia post was not selected, as it is difficult to fabricate and expensive. ${ }^{3}$

To achieve maximum esthetic, an ideal pontic should emerge from gingiva, support soft tissue and adjacent papillae. To manage ridge defect, several approaches of pontic site development are available. Among all the techniques, Abraham technique of palatal flap was used, as blood supply is maintained and it is a more conservative procedure. ${ }^{5}$ Immediate provisional restoration with ovate pontic design helped in developing the pontic site. ${ }^{8}$

All ceramic restorations are trending in recent dentistry, as it has better esthetic advantage over porcelain metal restoration. ${ }^{9}$ However, using such restoration over the metal core gives grayish hue to final restoration. The metal core needed to be mask to enhance the esthetic. Metal core can be masked either with composite or ceramic. ${ }^{10}$ Composite requires specialized metal primer for adherence of it to metal core, whereas in case of ceramic, metal core needs to be sandblasted to create a rough surface. Thus, a ceramic opaque was used for masking the all-ceramic restoration that enhanced the esthetic of final restoration.

\section{CONCLUSION}

Today, the demand for all-ceramic restoration is increasing. But such restorations are difficult to use in cases wherein there is underlying metallic structure that will affect the esthetic value of all-ceramic restoration. This article presented a technique to mask the metallic hue of cast post to restore teeth with all-ceramic restoration giving a life-like appearance to restoration. Thus, a little modification gives better results and helps in achieving the desired esthetic output.

\section{REFERENCES}

1. Smith CT, Schuman N. Prefabricated post-and-core systems: an overview. Compend Contin Educ Dent 1998 Oct; 19(10):1013-1020.

2. Patel SS, Shah DN. Restoration options with post and core: review article. J Ahmedabad Dent Coll Hosp 2013;4(1):10-14.

3. Jotkowitz A, Samet N. Rethinking ferrule-a new approach to an old dilemma. Br Dent J 2010 Jul 10;209(1):25-33.

4. Galhano GA, Melo RM, Pavanelli CA, Baldissara P, Scotti R, Valandro LF, Bottino MA. Adhesive cementation of zirconia posts to root dentin: evaluation of the mechanical cycling effect. Braz Oral Res 2008 Jul-Sep;22(3):264-269.

5. Abrams L. Augmentation of the deformed residual edentulous ridge for fixed prosthesis. Compend Contin Educ Gen Dent 1980 May-Jun;1(3):205-213.

6. Chu SJ. Dental colour matching instruments and systemreview of clinical and research aspects. J Dent 2010;38 (Suppl 2): e2-e16.

7. Mezzomo E, Massa F, Libera SD. Fracture resistance of teeth restored with cast post and core designs cemented with two different cements. Quintessence Int 2003 Apr:34(4):301-306.

8. Liu CL. Use of a modified ovate pontic in area defects: a report of two cases. J Esthet Restor Dent 2004;16(5):273-283.

9. Raigrodski A. Concepts of design for contemporary anterior all ceramic restorations. J Cosm Dent 2013;28:46-58.

10. Vlahova A, Kissov, Kazakova R, Popova and Todorov G. Masking the metal color of cast post and core restoration by metal ceramic caps: a clinical report. JSM Dent 2014;2(2):1024. 